\title{
Terlipressin for Hepatorenal Syndrome-Acute Kidney Injury in Acute-On-Chronic Liver Failure: A Single-Center Retrospective Cohort Study
}

songtao liu

Beijing YouAn Hospital

Qinghua Meng

Beijing YouAn Hospital

Zhongjie Hu

Beijing YouAn Hospital

Jun Lu

Beijing YouAn Hospital

Mei Liu

Beijing YouAn Hospital

Meiping Wang

Capital Medical University

Yuan Xu

Beijing Tsinghua Chang Gung Hospital

Jianxin Zhou ( $\square$ zhoujx.cn@icloud.com )

Beijing Tian Tan Hospital

\section{Research Article}

Keywords: acute-on-chronic liver failure, hepatorenal syndrome, acute kidney injury, terlipressin, albumin

Posted Date: January 21st, 2021

DOl: https://doi.org/10.21203/rs.3.rs-143931/v1

License: (1) (1) This work is licensed under a Creative Commons Attribution 4.0 International License. Read Full License 


\section{Abstract}

Objectives: Patients with hepatorenal syndrome-acute kidney injury (HRS-AKI) based on acute-on-chronic liver failure (ACLF) have a high rate of mortality. Terlipressin combined with albumin is the first line therapy for HRS-AKI in cirrhosis, however, whether it is more effective than albumin alone in ACLF is unclear. We aimed to analyze therapeutic effects of terlipressin in these patients.

Methods: A retrospective study was conducted on patients with HRS-AKI based on ACLF at Beijing You'an Hospital between October 2010 and October 2019. The primary endpoint was a reversal rate of HRS-AKI, which was defined as serum creatinine $(\mathrm{sCr})$ reduced to within $0.3 \mathrm{mg} / \mathrm{dL}$ of baseline. The secondary endpoints included a reversal rate of HRS in patients with $\mathrm{sCr}<1.5 \mathrm{mg} / \mathrm{dL}$ and 28 -day survival.

Results: 221 of 755 patients meet inclusion criteria. 113 cases were treated with terlipressin plus albumin (TA group), whereas 108 cases with albumin alone (AA group). The reversal rate of HRS-AKI in TA group was higher compared to in AA group (35.39\% vs $23.15 \%, P=0.046)$. Additionally, reversal time in TA group was $8.18 \pm 4.39$ days, compared to $11.29 \pm 3.67$ days in AA group $(P=0.005)$. In patients with $\mathrm{sCr}$ $<1.5 \mathrm{mg} / \mathrm{dL}$, reversal rate of HRS-AKI in TA group was $41.46 \%(17 / 47)$ compared to $19.61 \%(10 / 51)$ in AA group $(P=0.067)$. Furthermore, reversal time in TA group was $8.12 \pm 5.75$ days, compared to $9.40 \pm 2.67$ days in AA group $(P=0.117)$. Notably, 28-day overall survival and transplantation-free survival in TA group was better than AA group $(P<0.001)$.

Conclusions: Terlipressin combined albumin was effective for HRS-AKI in ACLF. Combination therapy tends to be more beneficial for patients with sCr level $<1.5 \mathrm{mg} / \mathrm{dL}$.

\section{Introduction}

Hepatorenal syndrome (HRS) is a common complication of advanced liver diseases, characterised by renal failure and major disturbances in circulatory function ${ }^{[1]}$. Its incidence was about $8 \% \varangle 40 \%[2-4]$, and associated with an especially poor prognosis. The diagnosis of HRS was revised by International Club of Ascites (ICA) in 2007 and 2015, respectively ${ }^{[5,6]}$. Under revised consensus recommendations, HRS was reclassified according to dynamic serum creatinine $(\mathrm{s} C \mathrm{r})$ measurement and refined as HRS-acute kidney injury (HRS-AKI). A new algorithm for management of HRS-AKI was proposed. Vasoconstrictors and albumin were recommended in Stage 2 and 3 HRS-AKI ${ }^{[6]}$. Terlipressin plus albumin has been considered as the first-line therapeutic option, whereas noradrenaline as an alternative strategy ${ }^{[7]}$. Though HRS-AKI exhibited no renal parenchymal injury according to diagnosis criteria, renal tubules had been damaged with presence of bile cast nephropathy $(B C N){ }^{[8]}$, especially in acute-on-chronic liver failure (ACLF) ${ }^{[9,10]}$.

ACLF was a group of clinical syndromes based on chronic liver diseases, accompanied by multiple organ failure, early death and high mortality ${ }^{[11]}$. However, its definition is debated ${ }^{[12-14]}$. For example, decompensated cirrhosis was excluded by Asian Pacific Association for the Study of the Liver (APASL). AKI only was a grading index in APASL. Yet AKI was a critical component according to the European 
Association for the Study of the Liver-Chronic Liver Failure (EASL-CLIF) Consortium based on EASL-CLIF Acute-on-Chronic Liver Failure in Cirrhosis (CANONIC) study ${ }^{[13]}$. The north American consortium for the study of end-stage liver disease (NASCELD) also emphasized renal function, recommending dialysis or renal replacement therapy ${ }^{[14]}$. According to CANONIC study, ACLF grade was a determinant of response to terlipressin plus albumin in HRS ${ }^{[15]}$. However, liver failure (total bilirubin [TBil] $>12 \mathrm{mg} / \mathrm{dL}$ ) occurred in $27 \%$ of patients. According to the APASL, terlipressin was superior to noradrenaline in the management of HRS-AKI in ACLF, where dosage of albumin was about $60 \mathrm{~g} / \mathrm{d}^{[16]}$. In addition, alcohol consumption, as a predominant cause of ACLF, was more common than reactivation of hepatitis B. Terlipressin was proposed to exert beneficial effects on mortality and renal function in patients with cirrhosis and HRS$A K{ }^{[17]}$. However, HRS based on ACLF was different from decompensated cirrhosis ${ }^{[18,19]}$. It is unclear if terlipressin plus albumin is superior to albumin alone in ACLF. To fill in gaps in knowledge, we would evaluate reversal rate of terlipressin plus albumin HRS-AKI based on ACLF. Currently, there is no unified diagnostic standard for ACLF, and TBil is an independent predictor for HRS reversal and longer survival in

terlipressin-treated patients ${ }^{[20,21]}$. Thus, diagnosis criteria of APASL are more valuable for clinical practice. We retrospectively analyzed HRS-AKI in the context of revised diagnostic criteria for HRS and compared therapeutic effects of terlipressin plus albumin to albumin alone.

\section{Methods And Materials}

\section{Study design}

A retrospective study was conducted at Beijing You'an Hospital Affiliated to Capital Medical University between October 2010 and October 2019. The study protocol was approved by the Ethic Committee of the Beijing You'an Hospital of Capital Medical University (LL-2019-177-K). this study was conformed to the ethical guidelines of the Declaration of Helsinki. Eligibility criteria for enrolment included patients diagnosed with HRS-AKI based on ACLF. Diagnostic criteria of HRS-AKI from the consensous of ICA were referenced after excluding other causes of $\mathrm{AKl}$; and when the patient was nonresponsive to volume expansion with intravenous albumin ${ }^{[6]}$. This study included patients who met diagnostic criteria of ACLF according to the APASL, defined as an acute hepatic insult in a patient with previously diagnosed or undiagnosed chronic liver diseases or cirrhosis, manifesting as jaundice (TBil $\geq 5 \mathrm{mg} / \mathrm{dL}$ ) and coagulopathy (international normalized ratio $[I N R] \geq 1.5$ ), complicated within 4 weeks by ascites and/or encephalopathy, and thus associated with high 28-day mortality. ${ }^{[12]}$ The informed consent for study was obtained from the participants.

The following patients were excluded: (1) diagnosed with malignant tumor, (2) age less than 18 years, (3) continuous renal replacement therapy (CRRT) at the time of diagnosis, (4) history of liver transplantation, (5) history of coronary artery diseases, (6) acute variceal bleeding, (7) history of chronic kidney diseases, (8) survival less than 3 days, (9) combined with human immunodeficient virus (HIV) infection, (10) incomplete clinical information, (11) use of terlipressin for less than 2 days, (12) use of other vasoactive drugs, and (13) history of chronic heart failure. 
Chronic liver failure consortium acute-on-chronic liver failure (CLIF-C ACLF) score, which was proposed to be superior to predict short-term mortality in patients with $\mathrm{ACLF}^{[22-24]}$, was calculated using the following formula: CLIF-C ACLFs $=10 \times(0.33 \times$ CLIF-Organ Failure [OF] $+0.04 \times$ Age $+0.63 \times \ln ($ WBC count $)-2)$.

\section{Data collection and definition}

Data were extracted from electronic medical record. All patients were subjected to detailed history, physical/laboratory examination, routine hematological tests, kidney and liver function tests at diagnosis. The use of terlipressin plus albumin or albumin alone was reviewed. The adverse events related to terlipressin were also reviewed. The 28-day survival status of each patient was analyzed.

Dynamic sCr and albumin measurement were defined at diagnosis, 3 to 4 day, 6 to 8 day, 9 to 12 day, 13 to 18 day as $0,3,7,10,14$ day, respectively. If there were repeated tests, the highest value was selected for data analysis. Reversal of HRS-AKI was defined as return of sCr to a value within $0.3 \mathrm{mg} / \mathrm{dL}$ at baseline. ${ }^{[6]}$ Two researchers jointly analyzed data and made a common judgment. If there was a dispute, the third researcher would make a final judgment.

\section{Primary and secondary endpoint}

The primary endpoint was to compare reversal of HRS-AKI. The secondary endpoints were reversal of HRS-AKI in patients with $\mathrm{s} C \mathrm{r}<1.5 \mathrm{mg} / \mathrm{dL}$ and 28-day survival. We aimed to determine predictors of survival and response to terlipressin.

\section{Statistical analysis}

Statistical Package for the Social Sciences (SPSS) software (version.23.0) was used to analyze all the characteristic values, and outcome data. Clinical and biochemical indices were expressed as either mean \pm SD or median (interquartile range; IQR). Qualitative variables were represented as frequencies. The independent $t$ test or Mann-Whitney rank sum test was utilized to analyze continuous variables; and Chisquare test was used to analyze the categorical data. Uni- and multivariate Cox as well as binary logistic regression models were employed to determine potential predictors of 28-day survival, mortality and treatment response, respectively. $P<.05$ was considered statistically significant.. The plots and survival curves were performed using GraphPad prism software (version 6.0).

\section{Results}

\section{Patient characteristics}

During this period, 755 ACLF with HRS-AKI patients were reviewed; 221 met inclusion criteria either at admission or during the course of hospitalization (Fig. 1). HBV infection and alcohol consumption were the most common acute and chronic insults. 113 patients were included in terlipressin plus albumin (TA) 
group and 108 in albumin alone (AA) group. Baseline demographic and clinical characteristics of patients were presented in Table 1. 
Table 1

Demographics and baseline clinical characteristics of all eligible included patients.

$\begin{array}{lll}\text { Variables } & \text { Terlipressin plus albumin (TA) group } & \text { Albumin alone (AA) group } P \text { value } \\ & (n=113) & (n=108)\end{array}$

\section{Demographic}

\begin{tabular}{|c|c|c|c|}
\hline Age, years & $52.43 \pm 11.93$ & $52.91 \pm 11.68$ & .77 \\
\hline Sex, M/F & $93 / 20$ & $88 / 20$ & .87 \\
\hline Hypertension, No. & 22 & 21 & .50 \\
\hline Diabetes mellitus, No. & 21 & 15 & .35 \\
\hline \multicolumn{4}{|l|}{ Etiology, No. } \\
\hline Alcohol & 47 & 40 & .49 \\
\hline HBV & 51 & 58 & .20 \\
\hline $\mathrm{HCV}$ & 3 & 2 & 1.00 \\
\hline PBC & 7 & 3 & .33 \\
\hline Others & 5 & 5 & 1.00 \\
\hline \multicolumn{4}{|l|}{ Infection, No. } \\
\hline SBP & 54 & 61 & .19 \\
\hline Pneumonia & 13 & 11 & .75 \\
\hline Others & 7 & 0 & - \\
\hline None & 39 & 36 & .85 \\
\hline WBC $\left(\times 10^{9} / L\right)$ & $8.77(6.33,12.80)$ & $9.59(6.21,14.05)$ & .59 \\
\hline $\mathrm{HB}(\mathrm{g} / \mathrm{dL})$ & $9.2(7.2,11.3)$ & $9.5(7.6,11.2)$ & .53 \\
\hline $\operatorname{PLT}\left(\times 10^{9} / \mathrm{L}\right)$ & $68.00(43.5,107.50)$ & $73.00(48.25,100.75)$ & .71 \\
\hline
\end{tabular}

Datas are presented as geometric mean \pm SD or median (interquartile range; IQR) or number.

$P$ value was obtained by Mann-Whitney $\mathrm{U}$ test, independent $\mathrm{t}$ test or Pearson chi-square tests when appropriate.

$\mathrm{PBC}=$ Primary biliary cholangitis; $\mathrm{SBP}=$ Spontaneous bacterial peritonitis; $\mathrm{WBC}=$ White blood cell count; $\mathrm{HB}=$ Hemoglobin concentration; $\mathrm{PLT}=$ Platelet count; $\mathrm{TBil}=$ Total bilirubin; $\mathrm{DBil}=$ = Direct bilirubin; $\mathrm{ALT}=$ Alanine transaminase; $\mathrm{AST}=$ Aspartate transaminases; $\mathrm{BUN}=\mathrm{Blood}$ urea nitrogen; $\mathrm{SCr}$ = Serum creatinine concentration; $\mathrm{Na}=$ Serum sodium concentration; $\mathrm{K}=$ Serum potassium concentration; INR = International normalized ratio; MAP = Mean arterial pressure; MELD = Model for End-stage Liver Disease score; CLIF-C ACLFs = Chronic liver failure consortium acute-on-chronic liver failure score. 


\begin{tabular}{|c|c|c|c|}
\hline Variables & $\begin{array}{l}\text { Terlipressin plus albumin (TA) group } \\
(n=113)\end{array}$ & $\begin{array}{l}\text { Albumin alone (AA) group } \\
(n=108)\end{array}$ & $P$ value \\
\hline TBil (mg/dL) & $24.00 \pm 12.86$ & $25.21 \pm 10.55$ & .44 \\
\hline $\mathrm{DBil}(\mathrm{mg} / \mathrm{dL})$ & $12.79(6.81,17.04)$ & $12.52(7.98,16.25)$ & .98 \\
\hline ALT (IU/L) & $41.50(22.40,84.75)$ & $44.80(22.45,108,23)$ & .51 \\
\hline AST (IU/L) & $82.70(55.75,143.40)$ & $95.70(57.30,165.95)$ & .43 \\
\hline Albumin $(\mathrm{g} / \mathrm{dL})$ & $2.88 \pm 0.52$ & $2.81 \pm 0.44$ & .35 \\
\hline BUN (mg/dL) & $13.40(9.96,20.04)$ & $12.75(8.40,17.33)$ & .09 \\
\hline $\mathrm{sCr}(\mathrm{mg} / \mathrm{dL})$ & $1.60(1.32,2.10)$ & $1.54(1.22,2.31)$ & .49 \\
\hline $\mathrm{sCr}<1.5 \mathrm{mg} / \mathrm{dL}$, No. & 47 & 51 & .42 \\
\hline $\mathrm{Na}(\mathrm{mmol} / \mathrm{L})$ & $130.11 \pm 6.30$ & $129.92 \pm 5.57$ & .81 \\
\hline $\mathrm{K}(\mathrm{mmol} / \mathrm{L})$ & $4.15 \pm 0.81$ & $4.19 \pm 0.89$ & .68 \\
\hline INR & $2.09(1.81,2.50)$ & $2.16(1.83,2.71)$ & .51 \\
\hline MAP (mmHg) & $83.00(78.00,92.00)$ & $83.00(75.50,92.75)$ & .79 \\
\hline MELD & $29.24 \pm 7.53$ & $30.12 \pm 6.42$ & .35 \\
\hline CLIF-C ACLFs & $46.28 \pm 7.09$ & $47.39 \pm 7.08$ & .24 \\
\hline \multicolumn{4}{|c|}{ Datas are presented as geometric mean \pm SD or median (interquartile range; IQR) or number. } \\
\hline \multicolumn{4}{|c|}{$\begin{array}{l}P \text { value was obtained by Mann-Whitney } \mathrm{U} \text { test, independent } \mathrm{t} \text { test or Pearson chi-square tests when } \\
\text { appropriate. }\end{array}$} \\
\hline \multicolumn{4}{|c|}{ 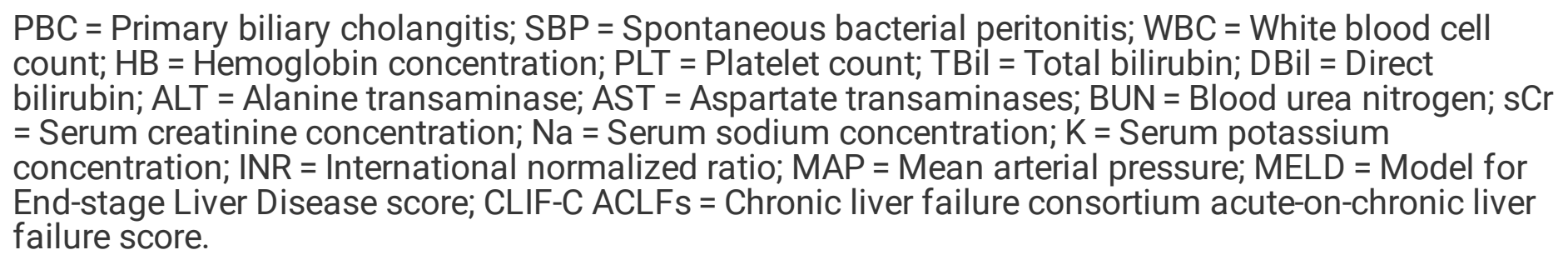 } \\
\hline
\end{tabular}

\section{The use of terlipressin and/or albumin in TA group and AA group}

In TA group, 85 patients were treated with terlipressin by continuous intravenous infusion. The initial dose of 76 patients was $2 \mathrm{mg} / \mathrm{d}$; 4 patients were adjusted to $3 \mathrm{mg} / \mathrm{d}$ whereas 2 patients to $4 \mathrm{mg} / \mathrm{d}$ on day 3 . The initial dose of 9 patients were $4 \mathrm{mg} / \mathrm{d}$, and 3 were adjusted to $2 \mathrm{mg} / \mathrm{d}$ on day 3.28 patients were administered by regular intravenous bolus injection at a dose of 2-4 mg/d, and 4 patients reduced dosage on day 3. The administration time of terlipressin was $7.18 \pm 3.91$ days. Total dosage of albumin in TA group vs. AA group was $132.79 \pm 57.51 \mathrm{~g}$ vs. $140.72 \pm 63.39 \mathrm{~g}(P=.331)$, respectively. The average 
dosage was $14.56 \pm 3.69 \mathrm{~g} / \mathrm{d}$ and $14.66 \pm 3.91 \mathrm{~g} / \mathrm{d}(P=.839)$, respectively. The dynamic measurements of albumin between two groups were significant different from those on day 3 (Fig. 2).

\section{Efficiency of terlipressin plus albumin for HRS-AKI in ACLF}

The sCr in TA group was significantly lower than AA group in about 7 days $(P=.032)$ (Fig. 3). In TA group, rate of HRS-AKI reversal was 35.39\% (40 of 113), compared to $23.15 \%$ (25 of 108) in AA group $(P=.046)$. In patients with $\mathrm{s} C r<1.5 \mathrm{mg} / \mathrm{dL}$ at initial treatment, $41.46 \%$ (17 of 47 ) were reversed in TA group, whereas $19.61 \%$ (10 of 51 ) in AA group ( $P=.067$ ). Among patients with $\mathrm{sCr} \geq 1.5 \mathrm{mg} / \mathrm{dL}, 34.84 \%$ (23 of 66 ) were reversed in TA group whereas $26.31 \%$ (15 of 57) in AA group $(P=.307)$. There was no statistical difference within each group (TA group: $P=.884$, AA group: $P=.409$ ) (Fig. 4).

The reversal time in TA Group was $8.18 \pm 4.39$ days, compared to $11.29 \pm 3.67$ days in AA group $(P=$ .005). For patients with $\mathrm{sCr}<1.5 \mathrm{mg} / \mathrm{dL}$ at initial treatment, reversal time was $8.12 \pm 5.75$ days in TA group, compared to $9.40 \pm 2.67$ days in AA group $(P=.117)$. Among patients with $\mathrm{sCr} \geq 1.5 \mathrm{mg} / \mathrm{dL}$, reversal time was $8.22 \pm 3.18$ days in TA group whereas $12.64 \pm 3.75$ days in AA group $(P=.001)$. There was no statistical difference within TA group $(P=.884)$, whereas a significant difference within AA group $(P=.036)$ (Fig. 5).

In TA group, 5 cases relapsed, 1 case was reversed after terlipressin therapy, 1 case was reversed after terlipressin combined with norepinephrine therapy, 2 cases were treated with CRRT, and 1 case was not reintervened. Two patients in AA group relapsed and received CRRT.

\section{Adverse events}

In TA group, 20 patients had diarrhea, 11 patients improved after treatment with loperamide and/or montmorillonite powder, 6 patients were stable, and 3 patients improved after reducing the dose of terlipressin. 6 patients presented abdominal pain, with mild symptoms. 4 patients with abdominal pain and diarrhea and given a lower drug dose. One patient had abdominal distension, and recovered spontaneously. 2 patients presented with acrocyanosis (at the end of hands and feet), receiving no special treatment. One patient had palpitations and discomfort, and one patient had right knee joint pain. The symptoms relieved spontaneously.

\section{Predictive Factors For The Reverse Of Hrs-aki In Aclf}

In multivariate binary logistic regression models, the absence of SBP (odds ratio [OR]: $2.11 ; 95 \% \mathrm{Cl}$ : 1.127-3.949; $P=.02$ ), TBil (OR; .997; 95\% Cl: .996 - .999; $P=.001)$ and CLIF-C ACLFs (OR: .955; 95\% Cl: $0.912-1.000 ; P=.049)$ were predictors for reverse in HRS-AKI (Table 2). 
Table 2

Univariate and multivariate analysis of Pretreatment predictive factors for reverse of HRS-AKI in ACLF.

\begin{tabular}{|c|c|c|c|c|}
\hline Variables & $\begin{array}{l}\text { reverse } \\
(n=65)\end{array}$ & $\begin{array}{l}\text { non-reverse } \\
(n=156)\end{array}$ & $\begin{array}{l}P \\
\text { value }\end{array}$ & $\begin{array}{l}P, \text { Regression } \\
(\mathrm{OR}, 95 \% \mathrm{Cl})\end{array}$ \\
\hline Age, years & $52.38 \pm 10.19$ & $52.78 \pm 12.41$ & .82 & \\
\hline Sex, M/F & $49 / 16$ & $132 / 24$ & .13 & \\
\hline Hypertension, No. & 9 & 34 & .20 & \\
\hline $\begin{array}{l}\text { Diabetes mellitus, } \\
\text { No. }\end{array}$ & 10 & 26 & 1.00 & \\
\hline \multicolumn{5}{|l|}{ Etiology, No. } \\
\hline Alcohol & 28 & 59 & .55 & \\
\hline HBV & 29 & 80 & .38 & \\
\hline $\mathrm{HCV}$ & 2 & 3 & .63 & \\
\hline PBC & 3 & 7 & 1.00 & \\
\hline Others & 3 & 7 & 1.00 & \\
\hline \multicolumn{5}{|l|}{ Infection, No. } \\
\hline SBP & 25 & 90 & .01 & $\begin{array}{l}.02(2.11,1.127- \\
3.949)\end{array}$ \\
\hline Pneumonia & 7 & 17 & 1.00 & \\
\hline Others & 3 & 4 & .42 & \\
\hline None & 30 & 45 & .02 & \\
\hline WBC $\left(\times 10^{9} / \mathrm{L}\right)$ & $9.38(5.85,10.76)$ & $9.02(6.69,14.08)$ & .06 & \\
\hline $\mathrm{HB}(\mathrm{g} / \mathrm{dL})$ & $9.40(7.30,112.00)$ & $9.40(7.40,112.00)$ & .67 & \\
\hline PLT $\left(\times 10^{9} / \mathrm{L}\right)$ & $\begin{array}{l}74.00(47.50 \\
108.00)\end{array}$ & $\begin{array}{l}70.00(47.00 \\
101.00)\end{array}$ & .72 & \\
\hline Tbil (mg/dL) & $20.10 \pm 10.22$ & $26.46 \pm 11.91$ & $<.001$ & $\begin{array}{l}.001(.997, .996 \\
-.999)\end{array}$ \\
\hline Dbil (mg/dL) & $9.70(5.13,14.94)$ & $13.44(8.77,16.87)$ & .004 & \\
\hline ALT (IU/L) & $\begin{array}{l}45.40(23.20 \\
67.05)\end{array}$ & $\begin{array}{l}40.85(22.33 \\
109.88)\end{array}$ & .47 & \\
\hline$(\mathrm{IU} / \mathrm{L})$ & $\begin{array}{l}83.60(56.80 \\
127.45)\end{array}$ & $\begin{array}{l}92.35(54.80, \\
167.68)\end{array}$ & .30 & \\
\hline
\end{tabular}




\begin{tabular}{|c|c|c|c|c|}
\hline Variables & $\begin{array}{l}\text { reverse } \\
(n=65)\end{array}$ & $\begin{array}{l}\text { non-reverse } \\
(n=156)\end{array}$ & $\begin{array}{l}P \\
\text { value }\end{array}$ & $\begin{array}{l}P, \text { Regression } \\
(\mathrm{OR}, 95 \% \mathrm{Cl})\end{array}$ \\
\hline Albumin (g/dL) & $2.83 \pm 5.02$ & $2.85 \pm 4.78$ & .81 & \\
\hline BUN (mg/dL) & $11.63(9.24,18.33)$ & $13.22(9.52,17.85)$ & .46 & \\
\hline $\mathrm{sCr}(\mathrm{mg} / \mathrm{dL})$ & $1.61(1.28,2.06)$ & $1.55(1.26,2.27)$ & .77 & \\
\hline $\mathrm{Na}(\mathrm{mmol} / \mathrm{L})$ & $130.67 \pm 5.90$ & $129.74 \pm 5.95$ & .29 & \\
\hline $\mathrm{K}(\mathrm{mmol} / \mathrm{L})$ & $4.18 \pm 0.85$ & $4.16 \pm 0.85$ & .88 & \\
\hline INR & $2.06(1.82,2.49)$ & $2.18(1.81,2.79)$ & .27 & \\
\hline MAP $(\mathrm{mmHg})$ & $\begin{array}{l}83.00(73.50 \\
90.00)\end{array}$ & $\begin{array}{l}83.50(78.00 \\
93.00)\end{array}$ & .06 & \\
\hline MELD & $27.56 \pm 5.59$ & $30.55 \pm 7.36$ & .004 & \\
\hline CLIF-C ACLFs & $44.55 \pm 6.51$ & $47.77 \pm 7.12$ & .002 & $\begin{array}{l}.05(.955, .912 \\
-1.000)\end{array}$ \\
\hline
\end{tabular}

\section{Predictive Factors For 28-day Overall And Transplantation- free Survival}

Liver transplantation was operated in 11 patients, 10 survived and 1 died. Of 221 patients, 24 patients discharged from hospital lost follow-up, while 133 patients died within 28 days. The 28-day overall survival rate was $46.08 \%$ (47 of 102) in TA group, significantly better than in AA group $(17.89 \%, 17$ of 95 ; $P<.001$ ) (Fig. 6A). Among non-liver transplant patients, 28-day survival rate in TA group was $41.94 \%$ (39 of 93), significantly higher than in AA group (16.13\%, 15 of 93; $P=.001)$ (Fig. 6B). In multivariate Cox regression models, TBil (Hazard ratio [HR], 1.001; 95\% Cl: 1.000-1.002; $P=.003)$, INR (HR,1.151; $95 \% \mathrm{Cl}$ : 1.037-1.277; $P=.008)$ and CLIF-C ACLFs (HR, 1.048; 95\% Cl: 1.022-1.075; $P<.001)$ were independent predictors of 28-day overall survival (Table 3). After excluding cases with liver-transplantation, in multivariate Cox regression models, TBil (HR, 1.001; 95\% Cl: 1.000-1.002; $P=.002)$, INR (HR, 1.175; 95\% Cl: 1.071-1.290; $P=.001)$ and CLIF-C ACLFs (HR, 1.050; 95\% Cl: 1.024-1.077; $P<.001)$ were independent predictors of 28-day transplantation-free survival (Table 3 ). 
Table 3

Predictor of 28-day overall survival and transplantation-free survival of patients with HRS-AKI in ACLF.

\begin{tabular}{|c|c|c|c|c|}
\hline \multirow[t]{2}{*}{ Variables } & \multicolumn{2}{|l|}{ Univariate } & \multicolumn{2}{|c|}{ Multivariate } \\
\hline & $\mathrm{HR}(95 \% \mathrm{Cl})$ & PValue & $\mathrm{HR}(95 \% \mathrm{Cl})$ & PValue \\
\hline \multicolumn{5}{|c|}{ 28-day overall survival } \\
\hline ALT (IU/L) & $1.001(1.000-1.002)$ & .004 & & \\
\hline AST (IU/L) & $1.001(1.000-1.002)$ & .013 & & \\
\hline TBil (mg/dL) & $1.001(1.001-1.002)$ & $<.001$ & $1.001(1.000-1.002)$ & .003 \\
\hline DBil (mg/dL) & $1.002(1.000-1.003)$ & .004 & & \\
\hline INR & $1.198(1.102-1.302)$ & $<.001$ & $1.151(1.037-1.277)$ & .008 \\
\hline MELD & $1.044(1.019-1.069)$ & .001 & & \\
\hline CLIF-C ACLFs & $1.060(1.035-1.085)$ & $<.001$ & $1.048(1.022-1.075)$ & $<.001$ \\
\hline \multicolumn{5}{|c|}{ 28-day transplantation-free survival } \\
\hline ALT (IU/L) & $1.002(1.001-1.002)$ & $<.001$ & & \\
\hline AST (IU/L) & $1.002(1.001-1.003)$ & .002 & & \\
\hline TBil (mg/dL) & $1.002(1.001-1.002)$ & $<.001$ & $1.001(1.000-1.002)$ & .002 \\
\hline DBil (mg/dL) & $1.002(1.000-1.003)$ & .005 & & \\
\hline INR & $1.211(1.120-1.310)$ & $<.001$ & $1.175(1.071-1.290)$ & .001 \\
\hline MELD & $1.055(1.029-1.082)$ & .001 & & \\
\hline CLIF-C ACLFs & $1.062(1.037-1.087)$ & $<.001$ & $1.050(1.024-1.077)$ & $<.001$ \\
\hline
\end{tabular}

\section{Discussion}

Epidemiologically, incidence of HRS-AKI in ACLF was 7-35\% according to the APASL ${ }^{[16,25-30]}$. Effective treatment was essential to improve prognosis. Terlipressin is a specific V1A receptor agonist, and able to counteract splanchnic vasodilation ${ }^{[31]}$. Meta-analysis had reported HRS reverse rate as $42.0 \%$ in response to terlipressin treatment ${ }^{[32]}$. In our study, reverse rate was $35.39 \%$ with terlipressin plus albumin, better than albumin alone for HRS-AKI in ACLF, consistent with previous studies ${ }^{[18,30]}$ and meta-analysis ${ }^{[33]}$. Previous study reported reverse rate as $40 \%$ based on intention-to-treat analysis and $43.13 \%$ based on per-protocol analysis ${ }^{[16]}$. This difference might be explained by significantly higher dosage of albumin $(60 \mathrm{~g} / \mathrm{L})^{[34]}$ than recommendation $(20-40 \mathrm{~g} / \mathrm{d}){ }^{[7]}$. In our study, the dose of albumin in each group was lower and might reduce therapeutic effect. Dynamic value of albumin in AA group was significantly lower 
than that in TA group starting from day 3. Terlipressin may also play an auxiliary role in increasing albumin concentration and colloid osmotic pressure by maintaining vascular tone. However, terlipressin can reduce cardiac output. Thus, it is necessary to evaluate whether albumin combined with terlipressin can further increase reversal rate on the basis of increasing albumin dose. In addition, alcohol consumption is the major causes of ACLF in this study. In our study, HBV activation and alcohol consumption were the most important predisposing factors. It was reported that absence of alcoholic hepatitis was a predictor of reduction in AKI stage with treatment of HRS but longer transplant-free survival $^{[21,35]}$. There are still contradictions in different studies, so large-scale prospective research is required.

In revised consensus recommendation of HRS, these patients should be treated when $\mathrm{sCr}$ is $\geq 1.5 \mathrm{mg} / \mathrm{dL}$, however, for AKI stage 1 and $\mathrm{s} C r<1.5 \mathrm{mg} / \mathrm{dL}$, no agreement has been reached ${ }^{[6]}$. Patients with $\mathrm{s} C r<1.5$ $\mathrm{mg} / \mathrm{dL}$ get benefit from combinatory therapy, compared with albumin alone. The reversal time tended to be shortened. ACLF was deteriorated rapidly, it is very important to adopt active treatment strategies, especially for $\mathrm{sCr}<1.5 \mathrm{mg} / \mathrm{dL}$. In TA group, reversal rate was similar when $\mathrm{s} C \mathrm{r}<1.5 \mathrm{mg} / \mathrm{dL}$, not supporting early intervention. If we consider duration required to monitor changes in $\mathrm{sCr}$, early intervention may have advantage. For reversed patients in AA group, duration required for reversal was extend with the increase in $\mathrm{SCr}$. In addition to inadequate dose of albumin, difficulty in treatment was increased. In another study, most benefits derived from down-staging of AKI were in AKI Stage 1 at initial diagnosis ${ }^{[35]}$. So early intervention is very important. When $\mathrm{s} C r \geq 1.5 \mathrm{mg} / \mathrm{dL}$, reversal rate of was similar, though combinatory therapy could significantly shorten reversal time. HRS-AKI is a special prerenal azotemia without pathological injury. However, BCN was more common in ACLF compared to decompensated cirrhosis ${ }^{[8,36,37]}$. TBil was the most important predictor for reverse ${ }^{[18,19,32]}$. Hyperbilirubinemia was a prerequisite for inclusion, the higher the $\mathrm{sCr}$ value, the greater the possibility of kidney pathological changes. Thus, close monitoring and early treatment are important options.

In addition to therapeutic effect, terlipressin could cause a series of complications. Our study also identified diarrhea and abdominal pain ${ }^{[20,32]}$. Giving terlipressin by continuous venous infusion could reduce global daily dose and rate of adverse effects, ${ }^{[38]}$ however, some patients had to withdraw because of severe adverse events. It is important to keep patients on treatment and minimize withdrawals due to adverse events ${ }^{[21]}$. Patients receiving more than 3 days of treatment were more likely to achieve reversal with terlipressin ${ }^{[39]}$. There was a significant difference in $\mathrm{sCr}$ value on day 7 after treatment. Other studies also reported that reversal of HRS usually occurred within the second week of treatment ${ }^{[40,41]}$. Thus, time matters more than dose.

From our study, combinatory therapy exhibits advantages in 28-day overall survival and transplantationfree survival. Previous meta-analysis demonstrated terlipressin associated with beneficial effects on mortality ${ }^{[17]}$. However, no advantage was identified from a prospective study ${ }^{[20]}$ or another metaanalysis ${ }^{[42]}$. Findings from our retrospective study require further evaluation. Because of high mortality, liver transplantation should be considered when patient's condition is difficult to reverse. MELD is a very 
important reference index for liver transplantation. In our study, CLIF-C ACLFs is more promising for clinical application.

Limitations of this study include its retrospective nature and the fact that treatment is not randomized. Also, the dosage of albumin was not enough in each group. To better evaluate potential response for terlipressin therapy and beneficial effects on patient's survival, further prospective multicenter studies should be carried out.

\section{Conclusion}

This retrospective study of 221 patients treated between 2010 and 2019 has shown that terlipressin plus albumin is superior to albumin alone in HRS-AKI with ACLF, yet reversal rate is reduced. When $\mathrm{sCr}<1.5$ $\mathrm{mg} / \mathrm{dL}$, therapeutic effects terlipressin is more obvious. Prospective study will be conducted, especially for patients with $\mathrm{sCr} \geq 1.5 \mathrm{mg} / \mathrm{dL}$. More effective drugs for prevention of HRS-AKI based on ACLF are urgently needed.

\section{Declarations}

\section{Ethic approval and consent to participate}

This study was approved by Ethics Committee of The Institutional Review Board (IRB) at Beijing You'an Hospital, Capital Medical University. (LL-2019-177-K)

\section{Consent for publication}

Not applicable.

\section{Availability of data and materials}

Data is available from the corresponding author upon reasonable request.

\section{Competing interests}

None.

\section{Funding}

This research was supported by Scientific Research Project of Beijing Youan Hospital, CCMU 2019; China Primary Health Care Foundation - Youan Foundation of Liver Disease and AIDS (BJYAYY-GG2019-01).

\section{Authors' contributions}

JXZ and QHM: study concept and design, manuscript revision. STL, ZJH and ML: wrote the main manuscript text, data collection; JL, YX and MPW: data analysis and interpretation; All authors read and 
approved the final manuscript.

\section{Acknowledgements}

Not applicable.

\section{Authors' information}

${ }^{1}$ Department of Severe Liver Disease, Beijing You'an Hospital, Capital Medical University, Beijing, China.

${ }^{2}$ Department of Oncology, Beijing You'an Hospital, Capital Medical University, Beijing, China.

${ }^{3}$ Department of Epidemiology and Health Statistics, School of Public Health, Capital Medical University, Beijing, China.

${ }^{4}$ Department of Critical Care Medicine, Beijing Tsinghua Chang Gung Hospital, Beijing, China.

${ }^{5}$ Department of Critical Care Medicine, Beijing Tiantan Hospital, Capital Medical University, Beijing, China.

\section{References}

1. Ginès P, Guevara M, Arroyo V, Rodés J. Hepatorenal syndrome. Lancet. 2003; 362(9398): 1819-27.

2. Bashir MH, Iqbal S, Miller R, Singh J, Mubarak G, Likhtshteyn M, et al. Management and outcomes of hepatorenal syndrome at an urban academic medical center: a retrospective study. Eur $\mathrm{J}$ Gastroenterol Hepatol. 2019; 31(12): 1545-9.

3. Jamil K, Huang X, Lovelace B, Pham AT, Lodaya K, Wan G. The burden of illness of hepatorenal syndrome (HRS) in the United States: a retrospective analysis of electronic health records. J Med Econ. 2019; 22(5): 421-9.

4. Schepke M, Appenrodt B, Heller J, Zielinski J, Sauerbruch T. Prognostic factors for patients with cirrhosis and kidney dysfunction in the era of MELD: results of a prospective study. Liver Int. 2006; 26(7): 834-9.

5. Salerno F, Gerbes A, Ginès P, Wong F, Arroyo V. Diagnosis, prevention and treatment of the hepatorenal syndrome in cirrhosis a consensus workshop of the International Ascites Club. Gut. 2007; 56(9):1310-8.

6. Angeli P, Ginès P, Wong F, Bernardi M, Boyer TD, Gerbes A, et al. Diagnosis and management of acute kidney injury in patients with cirrhosis: revised consensus recommendations of the International Club of Ascites. J Hepatol. 2015; 62(4): 968-74.

7. European Association for the Study of the Liver. EASL clinical practice guidelines for the management of patients with decompensated cirrhosis. J Hepatol. 2018; 69(2): 406-60. 
8. van Slambrouck CM, Salem F, Meehan SM, Chang A. Bile cast nephropathy is a common pathologic finding for kidney injury associated with severe liver dysfunction. Kidney Int. 2013; 84(1): 192-7.

9. Nayak SL, Kumar M, Bihari C, Rastogi A. Bile cast nephropathy in patients with acute kidney injury due to hepatorenal syndrome: a postmortem kidney biopsy study. J Clin Transl Hepatol. 2017; 5(2): 92-100.

10. Maiwall R, Kumar S, Chandel SS, Guresh Kumar G, Archana Rastogi A, Chhagan Bihari C, et al. AKI in patients with acute on chronic liver failure is different from acute decompensation of cirrhosis. Hepatol Int. 2015; 9(4): 627-39.

11. Anand AC, Dhiman RK. Acute on chronic liver failure-what is in a 'Definition'? J Clin Exp Hepatol. 2016; 6(3): 233-40.

12. Sarin SK, Choudhury A, Sharma MK, Maiwall R, Al Mahtab M, Rahman S, et al. Acute-on-chronic liver failure: consensus recommendations of the Asian Pacific association for the study of the liver (APASL): an update. Hepatol Int. 2019;13(4):353-90.

13. Moreau R, Jalan R, Gines P, Pavesi M, Angeli P, Cordoba J, et al. Acute-on-chronic liver failure is a distinct syndrome that develops in patients with acute decompensation of cirrhosis. Gastroenterology. 2013; 144(7): 1426-37, 1437, e1421-29.

14. Bajaj J, O'Leary J, Reddy K, Wong F, W,Biggins S, Patton H, et al. Survival in sepsis-related acute-onchronic liver failure is defined by extrahepatic organ failures. Hepatology. 2014; 6(1): 250-6.

15. Piano S, Schmidt HH, Ariza X, Amoros A, Romano A, Hüsing-Kabar A, et al. Association between grade of acute on chronic liver failure and response to terlipressin and albumin in patients with hepatorenal syndrome. Clin Gastroenterol Hepatol. 2018; 16(11): 1792-1800, e3.

16. Arora V, Maiwall R, Rajan V, Jindal A, Muralikrishna Shasthry S, Kumar G, et al. Terlipressin is superior to noradrenaline in the management of acute kidney injury in acute on chronic liver failure. Hepatology. 2020; 71(2): 600-10.

17. Allegretti AS, Israelsen M, Krag A, Jovani M, Goldin AH, Schulman AR, et al. Terlipressin versus placebo or no intervention for people with cirrhosis and hepatorenal syndrome. Cochrane Database Syst Rev. 2017; 6(6): CD005162.

18. Jiang QQ, Han MF, Ma K, Chen G, Wan XY, Kilonzo SB, et al. Acute kidney injury in acute-on-chronic liver failure is different from in decompensated cirrhosis. World J Gastroenterol. 2018; 24(21): 230010.

19. Davenport A, Sheikh MF, Lamb E, Agarwal B, Jalan R. Acute kidney injury in acute-on-chronic liver failure: where does hepatorenal syndrome fit? Kidney Int. 2017; 92(5): 1058-70.

20. Boyer TD, Sanyal AJ, Wong F, Frederick RT, Lake JR, O'Leary JG, et al. Terlipressin plus albumin is more effective than albumin alone in improving renal function in patients with cirrhosis and hepatorenal syndrome type 1. Gastroenterology. 2016; 150(7): 1579-89, e2.

21. Sanyal AJ, Boyer TD, Frederick RT, Wong F, Rossaro L, Araya V, et al. Type 1 reversal of hepatorenal syndrome type 1 with terlipressin plus albumin vs. placebo plus albumin in a pooled analysis of the 
OT-0401 and REVERSE randomised clinical studies. Aliment Pharmacol Ther. 2017; 45(11): 1390402.

22. Jalan R, Saliba F, Pavesi M, Amoros A, Moreau R, Ginès $P$, et al. Development and validation of a prognostic score to predict mortality in patients with acute-on-chronic liver failure. J Hepatol. 2014; 61(5): 1038-47.

23. Barosa R, Roque Ramos L, Nunes G, Jorge Fonseca J. CLIF-C ACLF score is a better mortality predictor than MELD, MELD-Na and CTP in patients with Acute on chronic liver failure admitted to the ward. Rev Esp Enferm Dig. 2017; 109(6): 399-405.

24. Engelmann C, Thomsen KL, Zakeri N, Sheikh M, Agarwal B, Jalan R, et al. Validation of CLIF-C ACLF score to define a threshold for futility of intensive care support for patients with acute-on-chronic liver failure. Crit Care. 2018; 22(1): 254.

25. Huang Z, Lin C, Fang J, Wang N, Zhou R, Pan C. Acute kidney injury in hepatitis B-related acute-onchronic liver failure without preexisting liver cirrhosis. Hepatol Int. 2015; 9(3): 416-23.

26. Maiwall R, Kumar S, Chandel SS, Kumar G, Rastogi A, Bihari C, et al. AKI in patients with acute on chronic liver failure is different from acute decompensation of cirrhosis. Hepatol Int. 2015; 9(4): 627-39.

27. Khatua CR, Panigrahi S, Mishra D, Pradhan S, Sahu SK, Barik RK, et al. Acute kidney injury at admission is a better predictor of mortality than its persistence at $48 \mathrm{~h}$ in patients with acute-onchronic liver failure. J Clin Transl Hepatol. 2018; 6(4): 396-401.

28. Maiwall R, Kumar G, Bharadwaj A, Jamwal K, Bhadoria AS, Jain P, et al. AKI persistence at 48h predicts mortality in patients with acute on chronic liver failure. Hepatol Int. 2017; 11(6): 529-39.

29. Yuan W, Zhang YY, Zhang ZG, Zou Y, Lu HZ, Qian ZP. Risk factors and outcomes of acute kidney injury in patients with hepatitis B virus-related acute-on-chronic liver failure. Am J Med Sci. 2017; 353(5): 452-8.

30. Jindal A, Bhadoria AS, Maiwall R, Sarin SK. Evaluation of acute kidney injury and its response to terlipressin in patients with acute-on-chronic liver failure. Liver Int. 2016; 36(1): 59-67.

31. Colson PH, Virsolvy A, Gaudard P, Charrabi A, Corbani M, Manière MJ, et al. Terlipressin, a vasoactive prodrug recommended in hepatorenal syndrome, is an agonist of human $\mathrm{V} 1, \mathrm{~V} 2$ and $\mathrm{V} 1 \mathrm{~B}$ receptors: Implications for its safety profile. Pharmacol Res. 2016; 113(Pt A): 257 - 64.

32. Wang H, Liu A, Bo W, Hu Y. Terlipressin in the treatment of hepatorenal syndrome: a systematic review and meta-analysis. Medicine (Baltimore). 2018; 97(16): e0431.

33. Jiang $W, H u Y$, Sun $Y$, Shen $Y, X u n$ Y. Prevalence and short-term outcome of acute kidney injury in patients with acute-on-chronic liver failure: A meta-analysis. J Viral Hepat. 2020; 27(8):810-817.

34. Lin S, Zhang DD, Gao Y, Zhu YK, Hou B. Letter to Editor: The synergistic effect of albumin on terlipressin in acute-on-chronic liver failure with acute kidney injury. Hepatology. 2019; 69(4):1845.

35. Wong F, Boyer TD, Sanyal AJ, Pappas SC, Escalante S, Jamil K. Reduction in acute kidney injury stage predicts survival in patients with type-1 hepatorenal syndrome. Nephrol Dial Transplant. 2020;35(9):1554-1561. 
36. Nayak SL, Kumar M, Bihari C, Rastogi A. Bile cast nephropathy in patients with acute kidney injury due to hepatorenal syndrome: a postmortem kidney biopsy study. J Clin Transl Hepatol. 2017; 5(2): 92-100.

37. Krones E, Eller K, Pollheimer MJ, Racedo S, Kirsch AH, Frauscher B, et al. NorUrsodeoxycholic acid ameliorates cholemic nephropathy in bile duct ligated mice. J Hepatol. 2017; 67(1): 110-9.

38. Cavallin M, Piano S, Romano A, Fasolato S, Frigo AC, Benetti G, et al. Terlipressin given by continuous intravenous infusion versus intravenous boluses in the treatment of hepatorenal syndrome: A randomized controlled study. Hepatology. 2016; 63(3): 983-92.

39. Sanyal AJ, Boyer T, Garcia-Tsao G, Regenstein F, Rossaro L, Appenrodt B, et al. A randomized, prospective, double-blind, placebo-controlled trial of terlipressin for type 1 hepatorenal syndrome. Gastroenterology. 2008; 134(5): 1360-8.

40. Moreau R, Durand F, Poynard T, Duhamel C, Cervoni JP, Ichaï P, et al. Terlipressin in patients with cirrhosis and type 1 hepatorenal syndrome: a retrospective multicenter study. Gastroenterology. 2002; 122(4): 923-30.

41. Solanki P, Chawla A, Garg R, Jain M, Sarin SK, et al. Beneficial effects of terlipressin in hepatorenal syndrome: a prospective, randomized placebo-controlled clinical trial. J Gastroenterol Hepatol. 2003; 18(2): 152-6.

42. Mary JT, Arthur T, Pratima S, Lok AS, Tapper EB. Limited Progress in Hepatorenal Syndrome (HRS) Reversal and Survival 2002-2018: A Systematic Review and Meta-Analysis. Dig Dis Sci. 2020; 65(5): 1539-48.

\section{Figures}




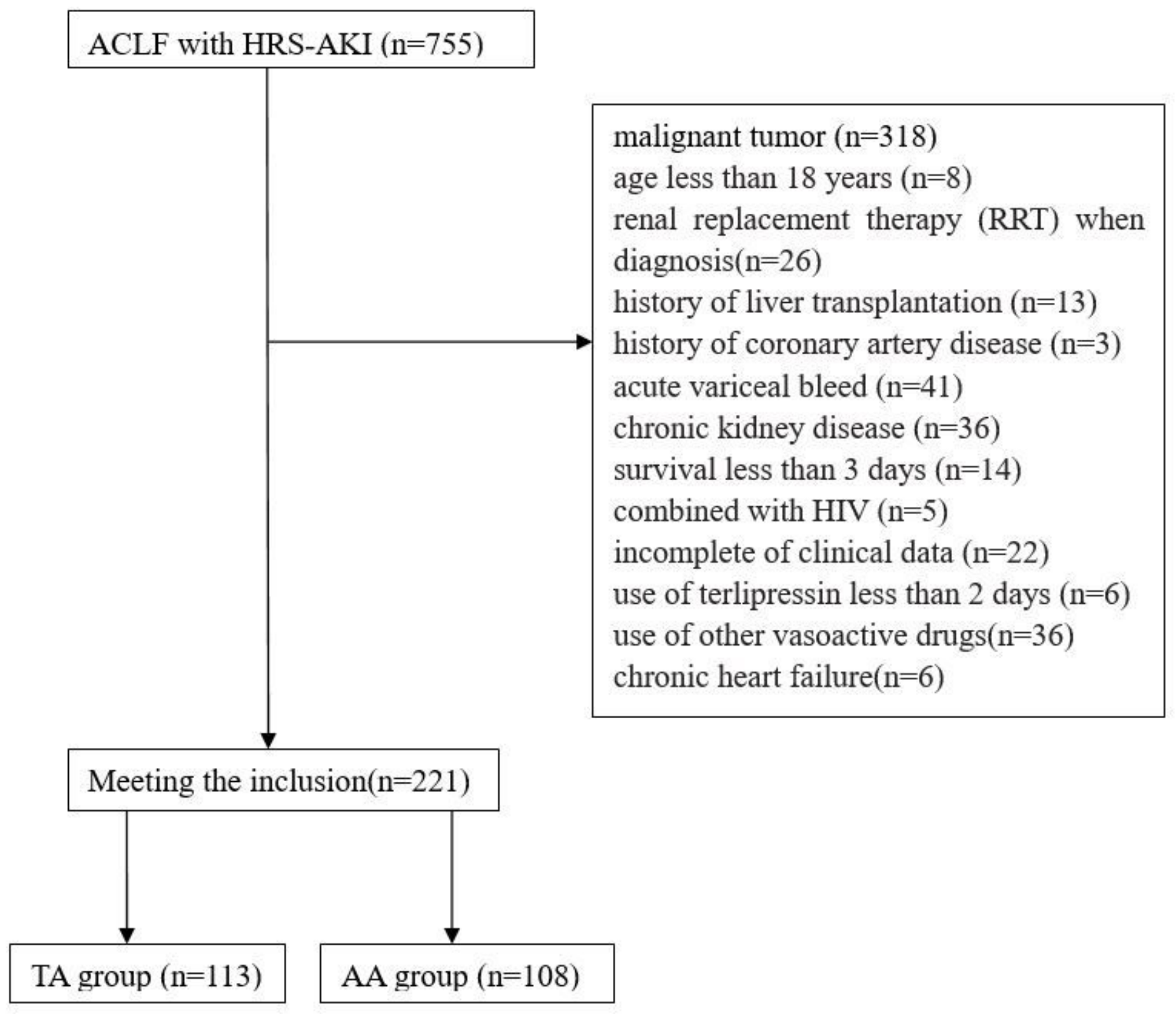

Figure 1

Flow chart of patient selection. 


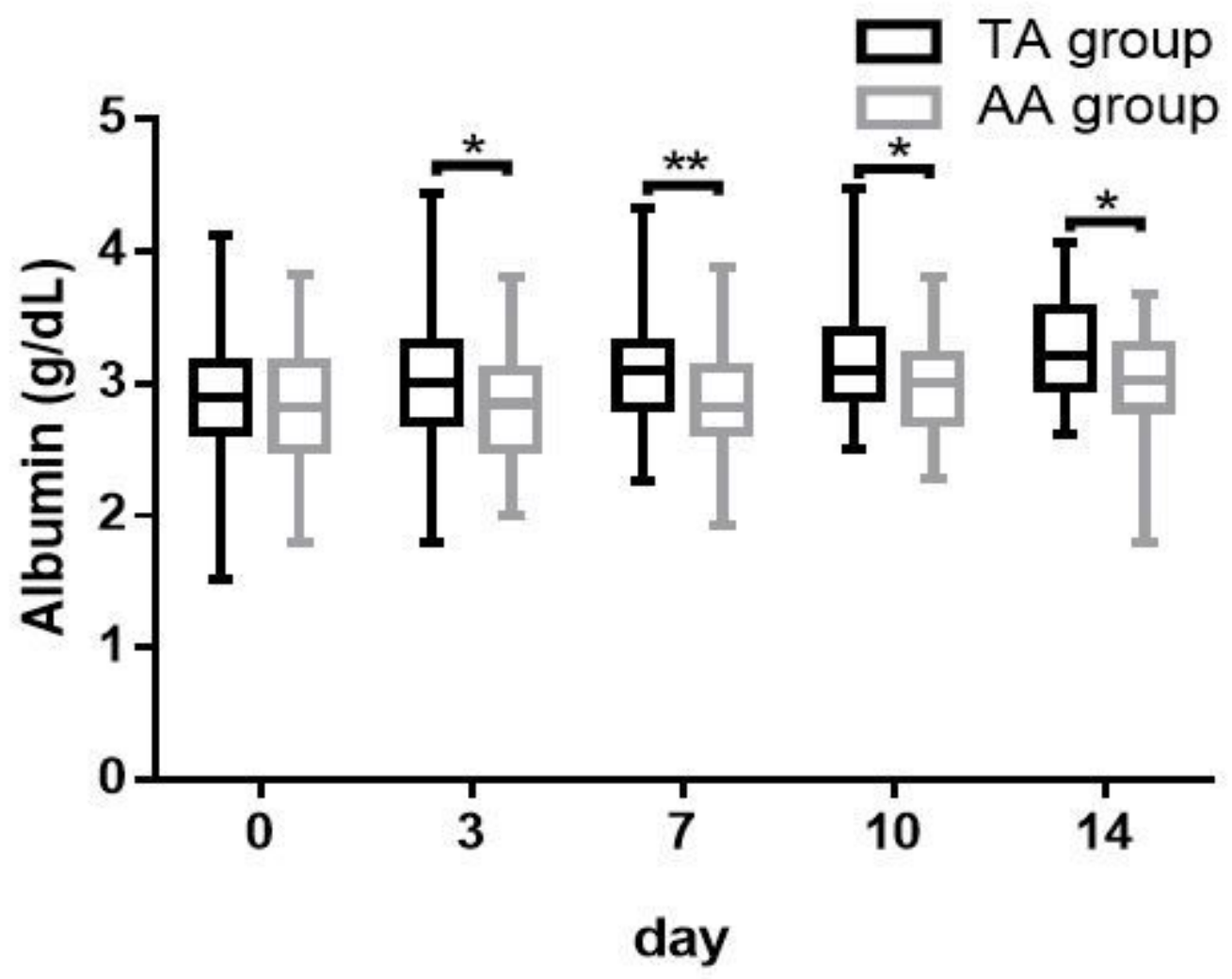

Figure 2

Dynamic tests of albumin in TA group vs. AA group ( $\left.{ }^{\star} P<0.05\right)$. In each column, plots represent mean (mix, max). $\mathrm{P}$ value was obtained by Mann-Whitney U-test. * means $\mathrm{P}<.05$, ** means $\mathrm{P}<.01$.

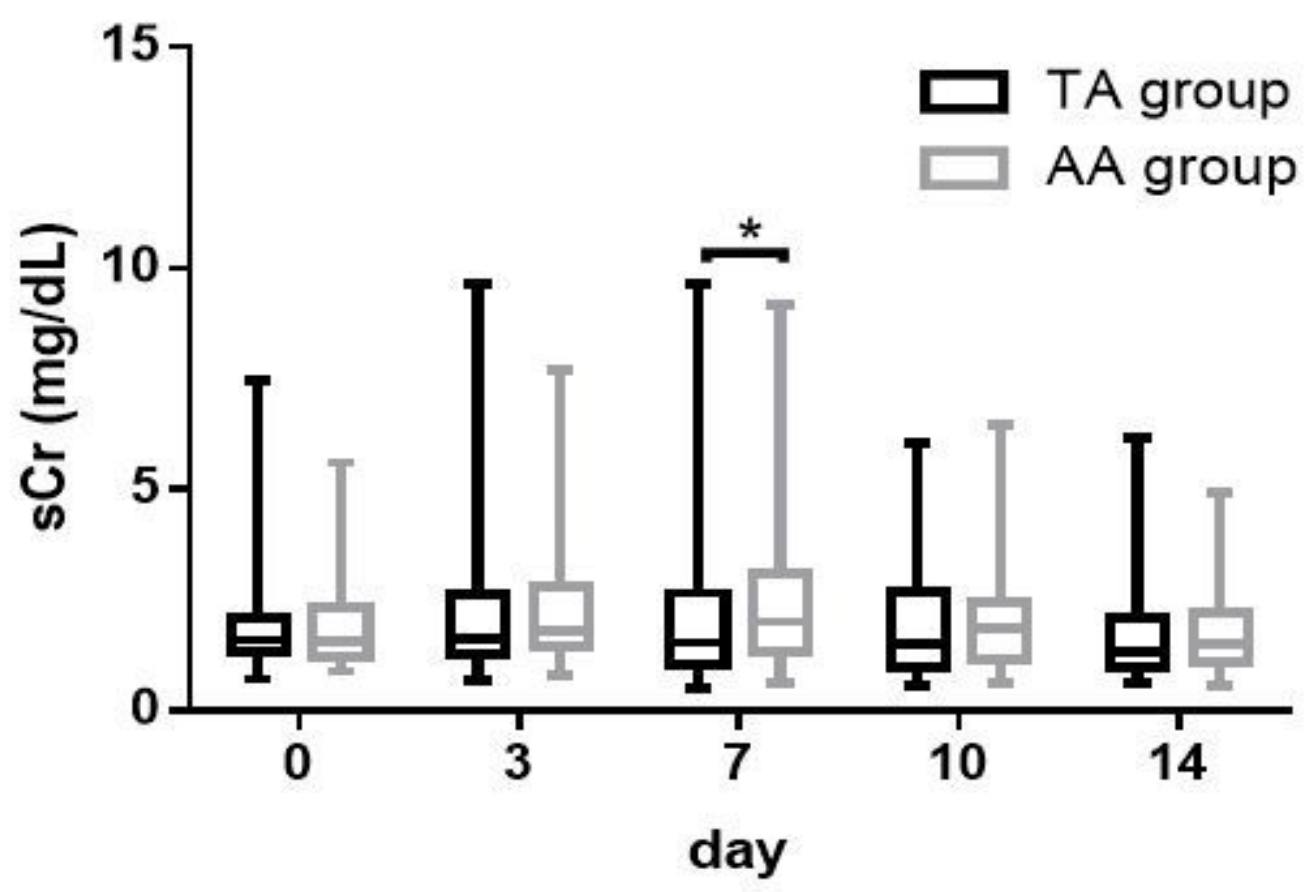


Figure 3

Dynamic tests of $\mathrm{sCr}$ in $\mathrm{TA}$ group vs. $\mathrm{AA}$ group. $\mathrm{sCr}$, serum creatinine concentration. In each column, plots represent mean (mix, max). P value was obtained by Mann-Whitney U-test. * means $\mathrm{P}<.05$.

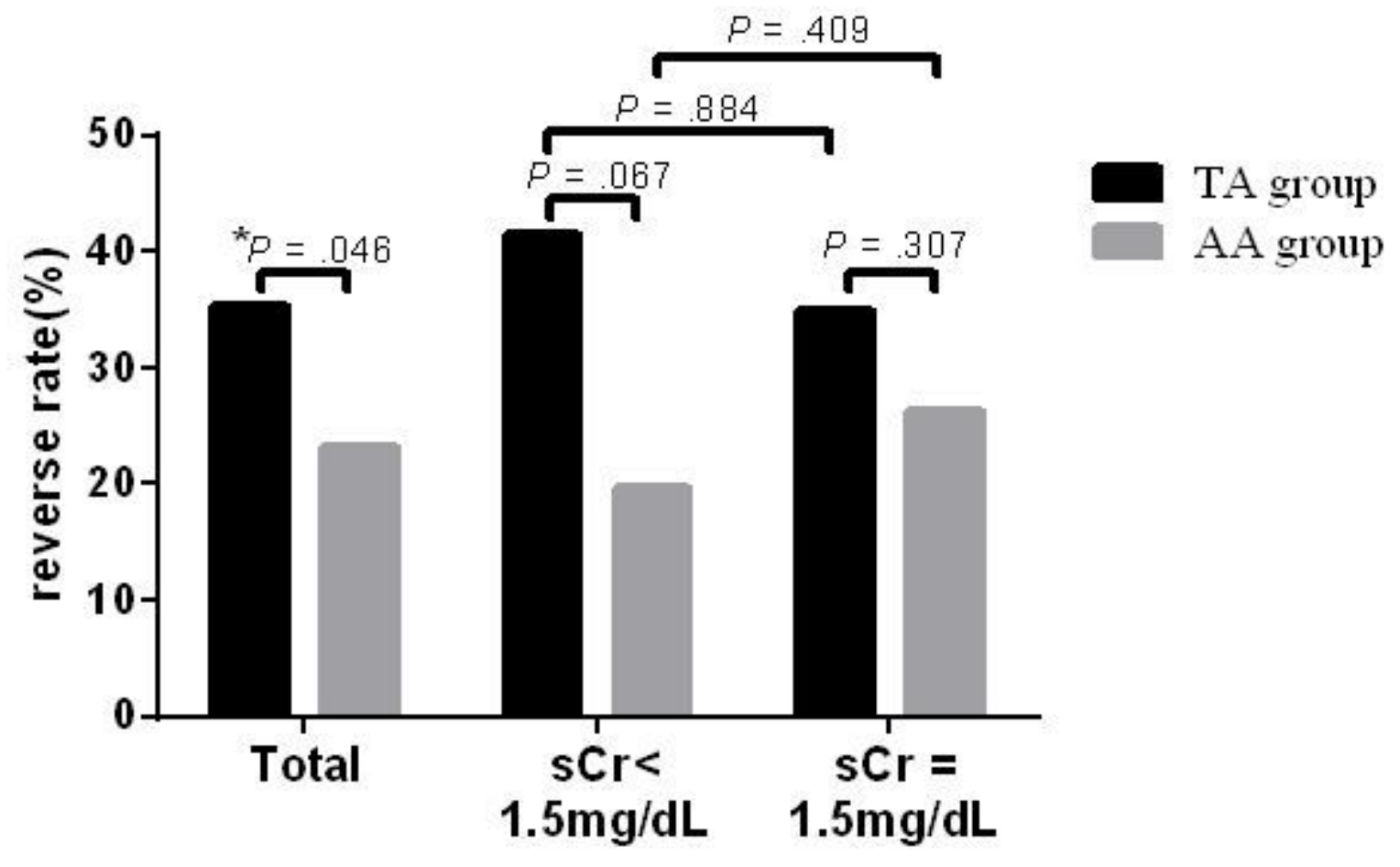

Figure 4

Different reversal rates of HRS-AKI between TA and AA group. $\mathrm{SCr}$, serum creatinine concentration. $\mathrm{P}$ value was obtained by Pearson chi-square test. * means $\mathrm{P}<.05$. 


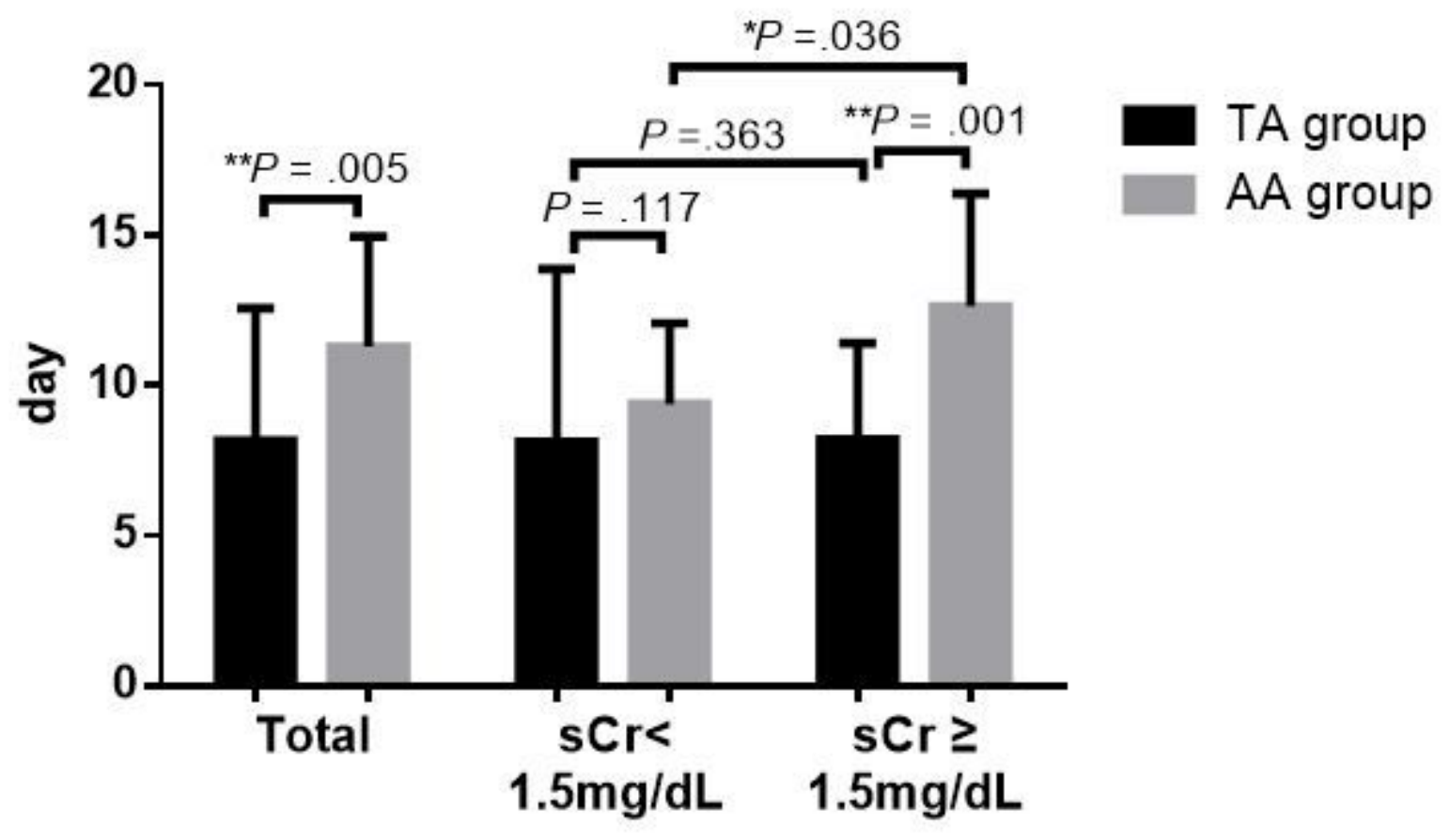

Figure 5

Different reversal time of HRS-AKI between TA and AA group. sCr, serum creatinine concentration. $P$ value was obtained by independent $t$ test. * means $\mathrm{P}<.05$, ** means $\mathrm{P}<.01$. 

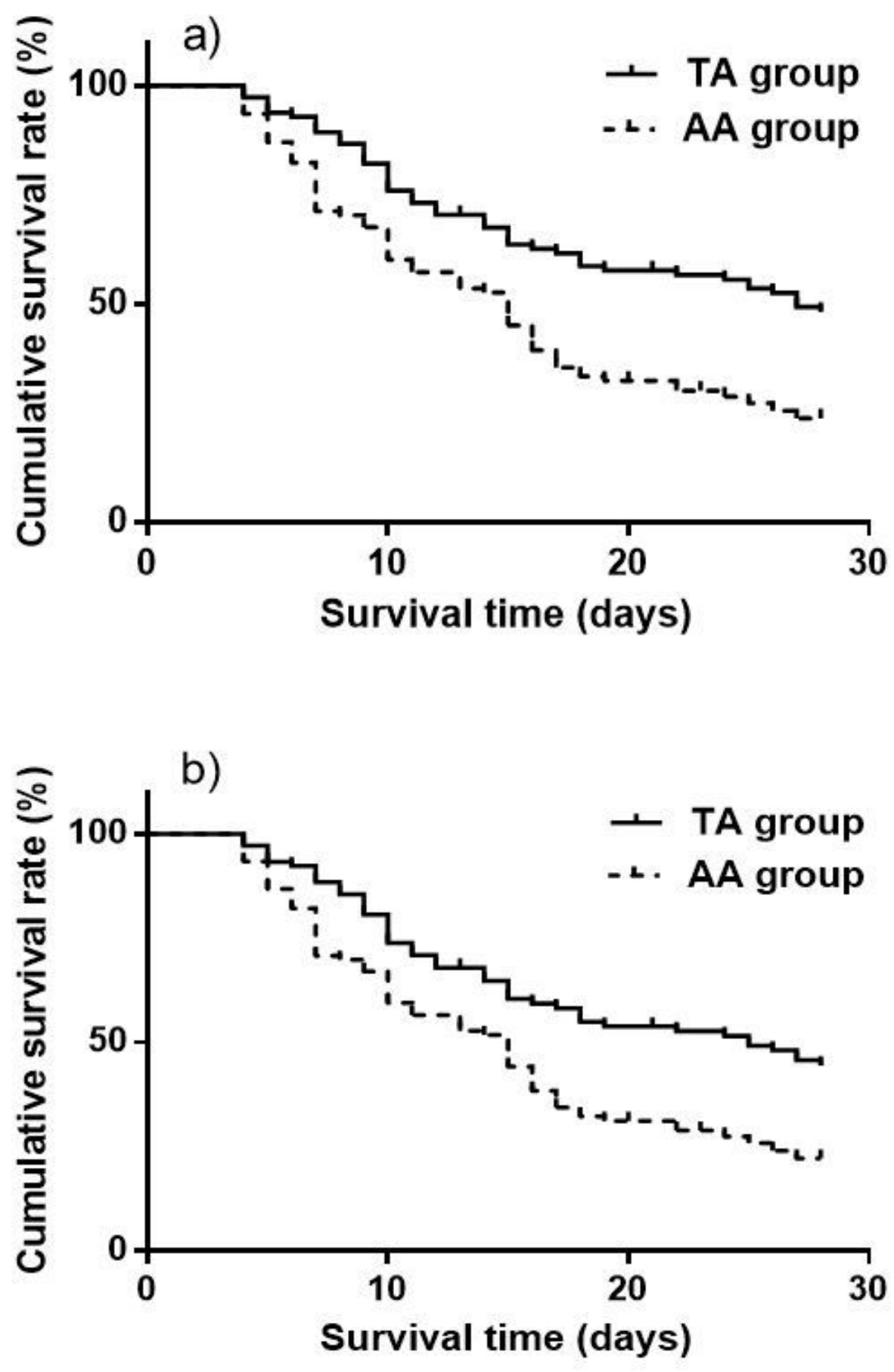

Figure 6

a) Comparison of 28-day overall cumulative survival rates between TA group and AA group using KaplanMeier plots $(P<.001)$. b) Comparison of 28-day transplantation-free cumulative survival rates between TA group and AA group using Kaplan-Meier plots $(P=.001)$ 